\title{
MINERAL INFORMATION EXTRACTION BASED ON GAOFEN-5'S THERMAL INFRARED DATA
}

\author{
Yao Liu ${ }^{1, *}$, Kun Shang ${ }^{1}$ \\ ${ }^{1}$ China Aero Geophysical Survey and Remote Sensing Center for Land and Resources, \\ Beijing, China, - yao.liu_agrs@foxmail.com, shangkun0213@126.com
}

KEY WORDS: Gaofen-5, thermal infrared, emissivity spectra, mineral information extraction, image simulation

\begin{abstract}
:
Gaofen-5 carries six instruments aimed at various land and atmosphere applications, and it's an important unit of China High-resolution Earth Observation System. As Gaofen-5's thermal infrared payload is similar to that of ASTER, which is widely used in mineral exploration, application of Gaofen-5's thermal infrared data is discussed regarding its capability in mineral classification and silica content estimation. First, spectra of silicate, carbonate, sulfate minerals from a spectral library are used to conduct spectral feature analysis on Gaofen-5's thermal infrared emissivities. Spectral indices of band emissivities are proposed, and by setting thresholds of these spectral indices, it can classify three types of minerals mentioned above. This classification method is tested on a simulated Gaofen-5 emissivity image. With samples acquired from the study area, this method is proven to be feasible. Second, with band emissivities of silicate and their silica content from the same spectral library, correlation models have been tried to be built for silica content inversion. However, the highest correlation coefficient is merely 0.592 , which is much lower than that of correlation model built on ASTER thermal infrared emissivity. It can be concluded that GF-5's thermal infrared data can be utilized in mineral classification but not in silica content inversion.
\end{abstract}

\section{INTRODUCTION}

Gaofen-5 (GF-5) satellite is the fifth flight unit of China Highresolution Earth Observation System (CHEOS) and will be launched in 2018. GF-5 will be carrying six types of instruments, including visible and short-wave infrared hyper-spectral camera, spectral imager, greenhouse gas detector, atmospheric environment infrared detector at very high spectral resolution, differential absorption spectrometer for atmospheric trace gas, and multi-angle polarization detector, with a designed lifespan of 8 years. Its thermal infrared (TIR) payload on the spectral imager will collect land surface thermal emission in four channels, which are centered at $8.20 \mu \mathrm{m}$ (TIR1), $8.63 \mu \mathrm{m}$ (TIR2), $10.80 \mu \mathrm{m}$ (TIR3) and $11.95 \mu \mathrm{m}$ (TIR4) with a spatial resolution of $40 \mathrm{~m}$ (Ye et al., 2017). Such spectral range settings are similar to the thermal infrared bands of ASTER instrument (see Figure 1).

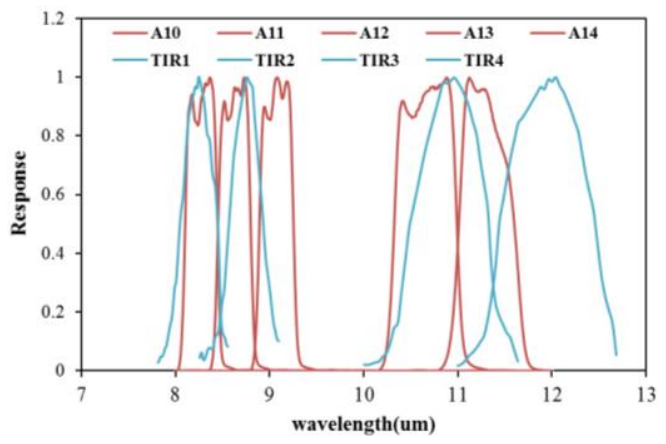

Figure 1. Spectral response functions of GF-5 TIR (TIR1-4) and ASTER TIR (A10-14) channels.

Previous research and applications show that ASTER TIR data has been successfully used to delineate mineral zones and assemblages of carbonate and silica (Kanlinowski et al., 2004). Specifically, sulfate, carbonate, and silicate minerals can be classified on ASTER images based on their difference of spectral features in thermal infrared bands (Ninomiya, et al., 2003). Also, the content of silica in silicate minerals can be estimated using ASTER TIR data; as silica is the main component of the earth's crust, and its content is an important chemical parameter for the classification of geological bodies and the analysis of its genetic evolution. Therefore, we see potentials of GF-5's thermal infrared data applied in mineral exploration.

In this paper, the ability of mineral classification and silica content estimation are analyzed using GF-5's thermal infrared bands, to prepare GF-5 thermal infrared data for possible application in mineral information extraction.

\section{STUDY AREA AND DATA}

\subsection{Study Area}

The study area is located about $15 \mathrm{~km}$ in the north of Liuyuan Town, which is located in the northwestern Gansu Province of China (see Figure 2). This area is in the Yujingzi and Liuyuan intracontinental rift zone, which is a polymetallic metallogenic belt that contains copper, gold, silver, iron, tungsten, molybdenum, lead and zinc (Cui et al., 2014).

\subsection{Data}

Since Gaofen-5 satellite has not been launched yet, emissivity image of GF-5's TIR bands at the study area is simulated for our research, and ASTER image was used as the simulation data source. This ASTER data was acquired on September 27, 2004.

In addition, to analyze minerals' spectral features indicated by GF-5's TIR data, carbonate, sulfate, and silicate mineral spectra from ASU (Arizona State University) spectral library is used. Specifically, 85 spectra of minerals from ASU emissivity spectral library are selected, including 7 carbonate samples, 5 sulfate samples, and 73 silicate samples (which contains 17 feldspars, 16

\footnotetext{
* Corresponding author
} 
amphiboles, 40 pyroxene). It is noted that carbonate, sulfate, and silicate comprise $1.7 \%, 0.1 \%$ and over $90 \%$ of earth crust's quality separately.

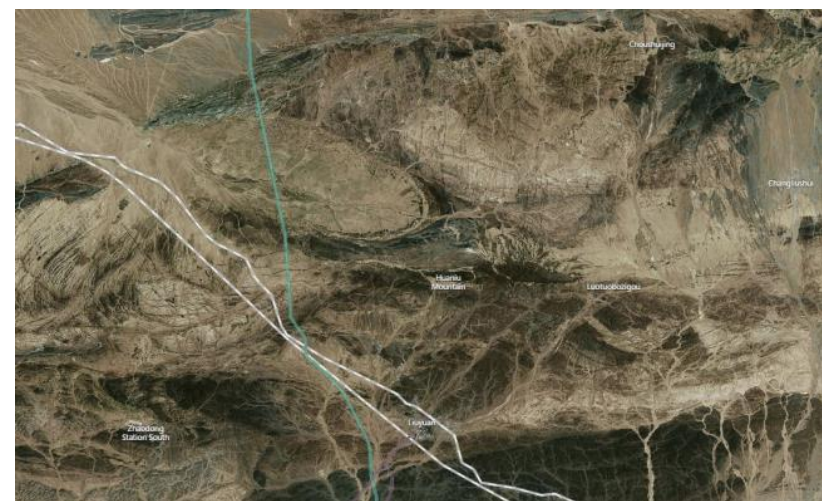

Figure 2. The map of the study area (@2018 Bing Maps)

\section{METHODOLOGY}

The basic processing steps applied for mineral classification and silica content estimation using GF-5 thermal infrared data are given in Figure 3 and explained in the following subsections briefly. First, band emissivities are calculated for these minerals with spectral response functions of GF-5's TIR bands. Then, spectral features of these minerals are analyzed based on GF-5's TIR band emissivities; spectral indices and correlation model are established for classification and silica content estimate, respectively. Finally, these established methods are performed on GF-5's emissivity images.

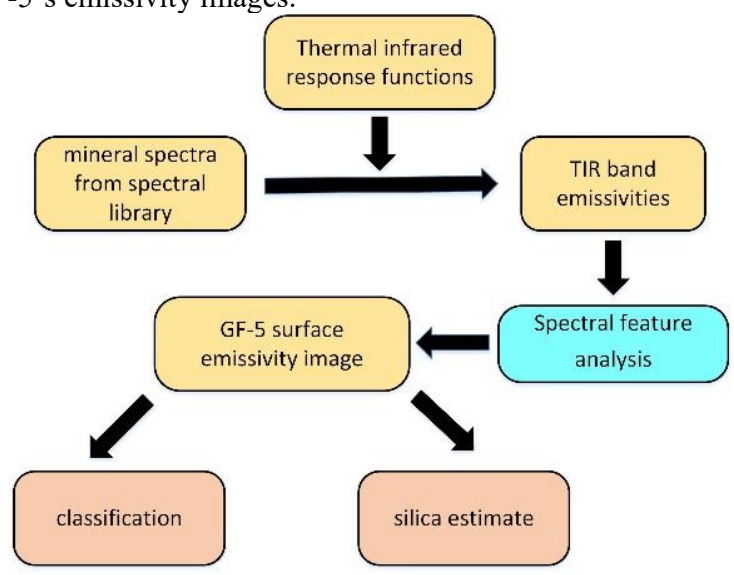

Figure 3. Flow chart used in the study.

\subsection{Spectral Indices}

We compared these minerals' TIR band emissivities, which are shown in Figure 4. It can be observed that carbonate minerals have a slightly higher emissivity in band TIR1 than in band TIR2, whereas sulfate and silicate minerals show the opposite spectral signature. Also, silicate and sulfate minerals have different spectral features in band TIR2, TIR3 and TIR4. That is, sulfate minerals have relatively higher emissivity in band TIR3 than in band TIR2 and TIR4, while silicates generally show absorption in TIR3 band emissivity. To quantitatively analyze these spectral features for classification, two spectral indices are designed, and they are defined as following:

$$
\mathrm{R} 1=\varepsilon_{2} / \varepsilon_{1}
$$

$$
\mathrm{R} 2=\left(\varepsilon_{2}+\varepsilon_{4}\right) / \varepsilon_{2}
$$

where $\quad \varepsilon_{i}=$ emissivity of $i$ th TIR band
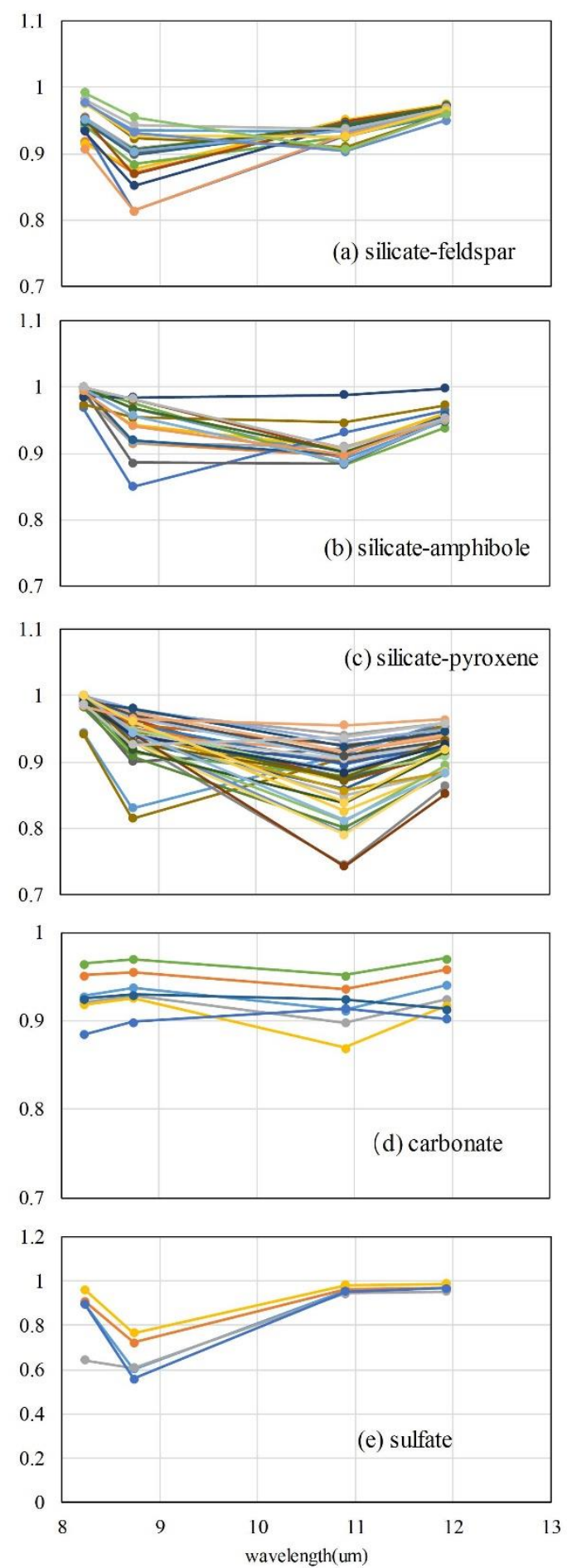

Figure 4. GF-5's TIR band emissivities of minerals in ASU library.

$\mathrm{R} 1$ and R2 are calculated for 85 spectra mentioned above and their range are listed in Table 1.

\begin{tabular}{|l|l|l|}
\hline Mineral Type & R1 range & R2 range \\
\hline carbonate & $1.003-1.015$ & - \\
\hline sulfate & $0.620-0.946$ & $0.799-0.893$ \\
\hline silicate & $0.865-0.999$ & $0.956-1.208$ \\
\hline
\end{tabular}

Table 1. Ranges of spectral indices from mineral in ASU library. 
From Table 1, we can see that $\mathrm{R} 1$ of carbonate minerals is greater than 1 , whereas sulfate and silicate minerals have an R1 of less than 1 . In addition, $\mathrm{R} 2$ of sulfate minerals ranges from 0.799 to 0.893 , and silicate minerals have a R2 of 0.956 to 1.208 . Therefore, by setting threshold values of R1 and R2, carbonate, sulfate, and silicate minerals can be classified based on Gaofen5's four thermal infrared band information as following:

- Carbonate: R1>1

- $\quad$ Sulfate: $\mathrm{R} 1<1$ and $\mathrm{R} 2<0.92$

- $\quad$ Silicate: $\mathrm{R} 1<1$ and R2 $>0.92$

\subsection{Correlation Analysis}

Each spectrum in ASU spectral library is accompanied by physical and compositional information. Therefore, silicate minerals' spectra in ASU library and their corresponding silica content can be used to build the content estimation model. Specifically, correlation analysis is conducted between GF-5's thermal infrared band emissivity of silicate minerals or spectra index calculated by these emissivities (i.e. $\varepsilon_{1} / \varepsilon_{2}, \varepsilon_{2} / \varepsilon_{3}, \varepsilon_{3} / \varepsilon_{4}$, $\left(\varepsilon_{1}+\varepsilon_{3}\right) / 2 \varepsilon_{2}$ and $\left.\left(\varepsilon_{2}+\varepsilon_{4}\right) / 2 \varepsilon_{3}\right)$, and the silica content; then band emissivity or spectral index with the highest correlation is selected. Finally, through regression analysis, the model with the highest correlation coefficient $\left(\mathrm{R}^{2}\right)$ will be finally chosen for silica content inversion. Correlation models with relatively high $\mathrm{R}^{2}$ are displayed in Figure 5 and listed in Table 2.
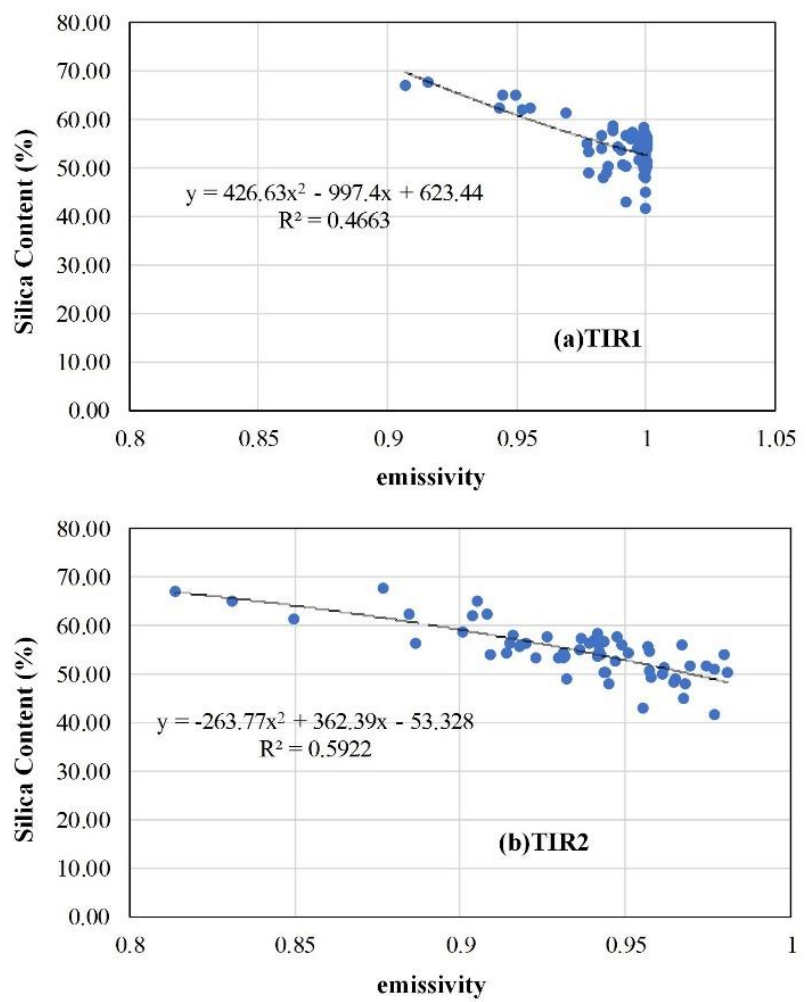
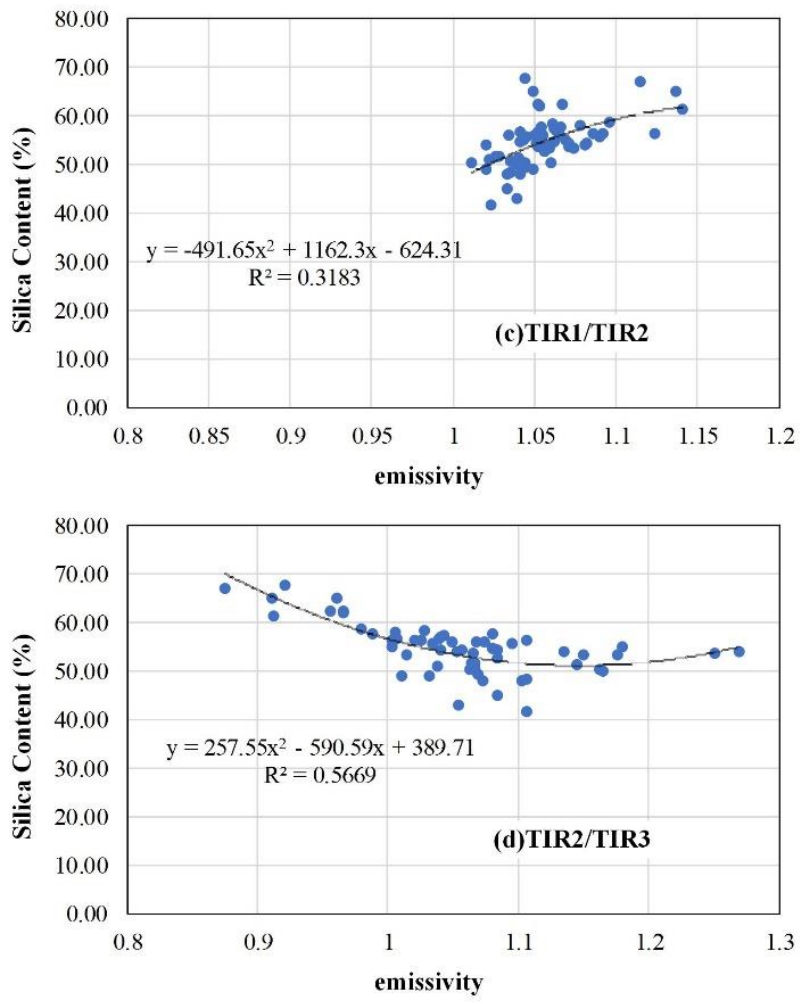

Figure 5 Correlation models established between GF-5's TIR band emissivity/spectral indices of silicate and silica content.

\begin{tabular}{|c|c|c|}
\hline $\begin{array}{c}\text { Emissivity/ } \\
\text { Spectra index }(\mathrm{x})\end{array}$ & $\begin{array}{c}\text { Correlation model } \\
\text { (y denotes silica content) }\end{array}$ & $\mathrm{R}^{2}$ \\
\hline$\varepsilon_{1}$ & $y=4.27 x^{2}-9.97 x+6.23$ & 0.466 \\
\hline$\varepsilon_{2}$ & $y=-2.64 x^{2}+3.62 x-0.53$ & 0.592 \\
\hline$\varepsilon_{1} / \varepsilon_{2}$ & $y=-4.92 x^{2}+11.62 x-6.24$ & 0.318 \\
\hline$\varepsilon_{2} / \varepsilon_{3}$ & $y=2.58 x^{2}-5.91 x+3.90$ & 0.567 \\
\hline
\end{tabular}

Table 2 Correlation model built for silica content estimate based on GF-5's TIR band emissivity/spectral index.

\section{RESULTS}

\subsection{Emissivity Image Generation}

The classification method is tested for our study area on GF-5's TIR simulated emissivity image, which is generated based on spectral mixing of ASTER data. The simulation method is similar to one that we've given in previous work (Liu et al., 2017). The simulated image is displayed in Figure 6.

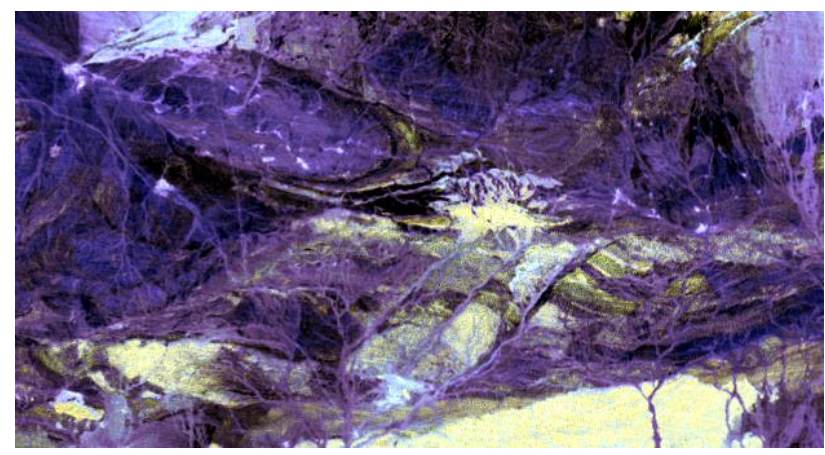


Figure 6. False color composite of simulated GF-5's TIR band emissivity image (R-TIR1, G-TIR2, B-TIR3).

\subsection{Result Analysis}

Mineral classification is conducted using thresholds of spectral indices proposed in Section 3.1. According to our experimental results, only carbonate and silicate minerals have been extracted; no sulfate minerals identified in the study area. We have collected 47 samples (14 carbonate and 33 silicate samples) in Liuyuan, and used these samples to validate the mapping results, which turn out to be with $94 \%$ and $100 \%$ correct of recognizing carbonate and silicate minerals, respectively. Moreover, through ground survey with geological information analysis, sulfate minerals do not exist in the study area.

According to the method proposed in Section 3.2, the correlation between TIR2 emissivity and the silica content was found to be the highest. The corresponding model is a quadratic polynomial one that's established based on TIR 2 emissivity. However, the model's coefficient of determination $\left(\mathrm{R}^{2}\right)$ is 0.592 , which is not very high. In contrast, silica content inversion model using ASTER data is a linear model based on a spectral index (the ratio of band 12 emissivity to that of band 13), with a $R^{2}$ of 0.78 . Concerning that linear model is more stable and with a higher $\mathrm{R}^{2}$ value, it can be inferred that GF-5's thermal infrared data is not as good as ASTER data for silica content inversion. Comparing the spectral range of GF-5 and ASTER TIR bands in Figure 1, we can see that ASTER's band 12 and 13 are basically overlapped by GF-5's TIR2 band, which shows that GF-5's band settings cannot exhibit spectral features of silica contents as that in ASTER data.

\section{CONCLUSIONS}

In this paper, the application of Gaofen-5's thermal infrared data is discussed for mineral information extraction, in terms of mineral classification and silica content estimate for silicate rocks. Based on mineral spectra from ASU spectral library, a classification method is proposed for carbonate, silicate and sulfate minerals, as well as the inversion model of silica content tried on correlation for silicate minerals. Since actual Gaofen-5 data is unavailable, the classification has been tested on simulated data, and accuracy analysis is conducted with ground data collected in the field. According to experimental results, using the method proposed in this paper, mineral classification can be performed using Gaofen-5's thermal infrared data. However, the silica content inversion model is not as good as that based on ASTER thermal data, showing that emissivity of GF-5's thermal infrared bands do not exhibit obvious spectral features associated with silica content.

\section{REFERENCES}

Ye X., Ren H., Liu R., Qin Q., Liu Y., Dong J., 2017. Land surface temperature estimate from Chinese Gaofen-5 satellite data using split-window algorithm. IEEE Transactions on Geoscience and Remote Sensing, vol. 55, no. 10, pp. 1-12.

Kanlinowski A., Oliver S., 2004. ASTER Mineral Index Processing. Remote Sensing Application Geoscience Australia. Australian Government Geoscience Website: http://www.ga.gov.au/image_cache/GA7833.pdf

Ninomiya, Y., Fu B., 2003. Extracting lithologic information from ASTER multispectral thermal infrared data in the northeastern Pamirs. Xinjiang Geology, vol. 21, no. 1, pp. 22-30.
Aboelkhair H., Ninomiya Y., Watanabe Y., Sato I., 2010. Processing and interpretation of ASTER TIR data for mapping of rare-metal-enriched albite granitoids in the central eastern desert of Egypt. Journal of African Earth Sciences, vol. 58, no. 1, pp. 141-151.

Cui J., Yan B., Wang R., Tian F., Zhao Y., Liu D., Yang S., Shen W., 2014. Regional-scale mineral mapping using ASTER VNIR/SWIR data and validation of reflectance and mineral map products using airborne hyperspectral CASI/SASI data. International Journal of Applied Earth Observation and Geoinformation, vol. 33, pp. 127-141.

Liu Y., Li N., Ren H. and Zhang T., 2017. Emissivity image simulation for thermal infrared bands on Gaofen-5 using airborne hyperspectral data, In Proc. IEEE International Geoscience and Remote Sensing Symposium (IGARSS), pp. 2093-2096. 